\title{
New Operational Matrix of Integrations and Coupled System of Fredholm Integral Equations
}

\author{
Hammad Khalil and Rahmat Ali Khan \\ Department of Mathematics, University of Malakand, Chakdara, Dir(L), Khyber Pakhtunkhwa, Dir Lower 23050, Pakistan \\ Correspondence should be addressed to Hammad Khalil; hammadk310@gmail.com
}

Received 7 December 2013; Accepted 31 December 2013; Published 13 February 2014

Academic Editors: Y. Feng and X.-1. Luo

Copyright ( $) 2014$ H. Khalil and R. A. Khan. This is an open access article distributed under the Creative Commons Attribution License, which permits unrestricted use, distribution, and reproduction in any medium, provided the original work is properly cited.

We study Legendre polynomials and develop new operational matrix of integration. Based on the operational matrix, we develop a new method to solve a coupled system of Fredholm integral equations of the form $U(x)+\lambda_{11} \int_{0}^{1} K_{11}(x, t) U(t) d t+$ $\lambda_{12} \int_{0}^{1} K_{12}(x, t) V(t) d t=f(x), V(x)+\lambda_{21} \int_{0}^{1} K_{21}(x, t) U(t) d t+\lambda_{22} \int_{0}^{1} K_{22}(x, t) V(t) d t=g(x)$, where $\lambda_{11}, \lambda_{12}, \lambda_{21}$, and $\lambda_{22}$ are real constants and $f, g \in C([0,1])$. The method reduces the coupled system to a system of easily solvable algebraic equations without discretizing the original system. As an application, we provide examples and numerical simulations demonstrating that the results obtained using the new technique match very well with the exact solutions of the problems. To show the efficiency of the method, we compare our results with some of the results already studied with other available methods in the literature.

\section{Introduction}

Fredholm integral equations are frequently encountered in many physical processes such as dynamic stiffness of rigid rectangular foundations [1], soil mechanics and rock mechanics [2], diffraction of waves by randomly rough surface in two dimensions [3], thermoelasticity [4], and scattering problem [5], to name a few. For systems of such equations, various techniques such as extrapolation method, Galerkin discretization, collocation methods, and quadrature, iterative, spline, orthogonal polynomial, and multiple grid methods have been proposed to determine desired solutions (see, e.g., [6-9] and the references quoted there). These methods include approximate analytical and numerical approaches.

Recently, approximate solutions to system of integral equations have attracted the attention of many authors and they obtained solutions using various available techniques in the literature. For example, system of integral equations has been studied with wavelets techniques in $[10,11]$, with Adomian decomposition method in [12,13], with Tau method in [14], with chebesheve polynomial and block pulse function in $[15,16]$, and with Taylor expansion and some modified methods based on taylor series expansion in [17-25].

In this paper, we use shifted Legendre polynomials and develop a new operational matrix of integration. Based on the operational matrix of integration, we develop a simple method to find solutions of the coupled system of Fredholm integral equations. The method reduces the coupled system to a system of easily solvable algebraic equations without discretizing the original system of equations. Besides simplicity, the method yields accurate results even for small value of $M$ resulting in the reduction of the system to small system of algebraic equations. It is verified by examples and their numerical simulations demonstrating that the results obtained using the new technique match very well with the exact solutions of the problems. To show the efficiency of the method over some of the well-known techniques, we compare our results with some of the results already studied with other available methods such as Taylor series approximation method [19] and block pulse method [16]. We find that the new techniques provide highly accurate solutions as compared to Taylor series approximation method and block pulse method. 


\section{Main Results: New Operational Matrix of Integrations}

The Legendre polynomials defined on $[-1,1]$ are given by the following recurrence relation:

$$
\begin{gathered}
\mathrm{Ł}_{i+1}(z)=\frac{2 i+1}{i+1} z £_{i}(z)-\frac{i}{i+1} £_{i-1}(z), \\
i=1,2, \ldots, \quad \text { where } \mathrm{Ł}_{0}(z)=0, \quad \mathrm{Ł}_{1}(z)=z .
\end{gathered}
$$

The transformation $x=(z+1) / 2$ transforms the interval $[-1,1]$ to $[0,1]$ and the polynomials transformed to the so called shifted Legendre polynomials given as [26] follows:

$$
P_{i}(x)=\sum_{k=0}^{i}(-1)^{i+k} \frac{(i+k) !}{(i-k) !} \frac{x^{k}}{(k !)^{2}}, \quad i=0,1,2,3, \ldots,
$$

where $P_{i}(0)=(-1)^{i}, P_{i}(1)=1$. The orthogonality condition is

$$
\int_{0}^{1} P_{i}(x) P_{j}(x) d x= \begin{cases}\frac{1}{2 i+1}, & \text { if } i=j \\ 0, & \text { if } i \neq j .\end{cases}
$$

Consequently, any $f(x) \in C[0,1]$ can be approximated by shifted Legendre polynomial as follows:

$$
f(x) \approx \sum_{a=0}^{m} c_{a} P_{a}(x)
$$

where $c_{a}=\left\langle f(x), P_{a}(x)\right\rangle=(2 a+1) \int_{0}^{1} f(x) P_{a}(x) d x$.

In vector notation, we write

$$
f(x)=K_{M}^{T} \widehat{P}_{M},
$$

where $M=m+1, K$ is the coefficient vector, and $\widehat{P}$ is $M$ terms vector function. In case of function of two variables, that is, $f \in C([0,1] \times[0,1])$, we write

$$
f(x, t) \approx \sum_{i=0}^{m} \sum_{j=0}^{m} c_{i j} P_{i}(x) P_{j}(t)
$$

where $c_{i j}=(2 i+1)(2 j+1) \iint_{0}^{1} f(x, t) P_{i}(x) P_{j}(t) d x d t$.

The orthogonality condition of $P_{i}(x) P_{j}(t)$ is found to be

$$
\begin{aligned}
& \iint_{0}^{1} P_{i}(x) P_{j}(t) P_{a}(x) P_{b}(t) d x d t \\
& \quad= \begin{cases}\frac{1}{(2 i+1)(2 j+1)}, & \text { if } a=i, b=j ; \\
0, & \text { otherwise. }\end{cases}
\end{aligned}
$$

In vector notation, (6) can be written as

$$
f(x, t) \approx\left(\widehat{P}_{M}(x)\right)^{T} C_{(M \times M)} \widehat{P}_{M}(t),
$$

where $\widehat{P}_{M}(x)$ and $\widehat{P}_{M}(t)$ are column vectors containing Legendre polynomial and $C$ is the coefficient matrix whose entries are obtained by using (6).
2.1. Error Analysis. For sufficiently smooth function $f(x, y)$ on $[0,1] \times[0,1]$, the error of the approximation is given by

$$
\left\|f(x, y)-P_{n}(x, y)\right\|_{2} \leq\left(C_{1}+C_{2}+C_{3} \frac{1}{M^{M+1}}\right) \frac{1}{M^{M+1}},
$$

where

$$
\begin{aligned}
& C_{1}=\frac{1}{4} \max _{(x, y) \in[0,1] \times[0,1]}\left|\frac{\partial^{M+1}}{\partial x^{M+1}} f(x, y)\right|, \\
& C_{2}=\frac{1}{4} \max _{(x, y) \in[0,1] \times[0,1]}\left|\frac{\partial^{M+1}}{\partial y^{M+1}} f(x, y)\right|, \\
& C_{3}=\frac{1}{16} \max _{(x, y) \in[0,1] \times[0,1]}\left|\frac{\partial^{2 M+2}}{\partial x^{M+1} \partial y^{M+1}} f(x, y)\right| .
\end{aligned}
$$

We refer the reader to [27] for the proof of the above result.

Lemma 1. Let $f(x, t) \in C([0,1] \times[0,1])$ and $g(t) \in C([0,1])$; then

$$
\int_{0}^{1} f(x, t) g(t) d t \approx K_{M} G_{M \times M} \widehat{P}(x),
$$

where $K_{M}$ is the Legendre coefficient vector of $g(t)$ and the matrix $G=\left[q_{j i}\right]$, where $q_{j i}=(1 /(2 j+1)) c_{i j}$.

Proof. In view of (5) and (6), we have

$$
f(x, t) \approx \sum_{i=0}^{m} \sum_{j=0}^{m} c_{i j} P_{i}(x) P_{j}(t),
$$

$$
\begin{gathered}
\text { where } c_{i j}=(2 j+1)(2 i+1) \iint_{0}^{1} f(x, t) P_{i}(x) P_{j}(t) d x d t \\
g(t) \approx \sum_{a=0}^{m} d_{a} P_{a}(t) \\
\text { where } d_{a}=(2 a+1) \int_{0}^{1} g(t) P_{a}(t) d t
\end{gathered}
$$

Using (12), we obtain

$$
\begin{aligned}
& \int_{0}^{1} f(x, t) g(t) d t \\
& \quad \approx \int_{0}^{1}\left(\sum_{i=0}^{m} \sum_{j=0}^{m} c_{i j} P_{i}(x) P_{j}(t)\right)\left(\sum_{a=0}^{m} d_{a} P_{a}(t)\right) d t
\end{aligned}
$$

which implies that

$$
\begin{aligned}
& \int_{0}^{1} f(x, t) g(t) d t \\
& \quad \approx \sum_{i=0}^{m} \sum_{j=0}^{m} \sum_{a=0}^{m} d_{a} c_{i j} P_{i}(x) \int_{0}^{1} P_{j}(t) P_{a}(t) d t .
\end{aligned}
$$


Using the orthogonality relation, we get

$$
\begin{aligned}
\int_{0}^{1} f(x, t) g(t) d t & \approx \sum_{i=0}^{m} \sum_{j=0}^{m} d_{j} c_{i j} P_{i}(x)\left(\frac{1}{2 j+1}\right) \\
& =\sum_{j=0}^{m} \sum_{i=0}^{m} d_{j} q_{j i} P_{i}(x),
\end{aligned}
$$

where $q_{j i}=(1 /(2 j+1)) c_{i j}$. In matrix form, we have

$$
\int_{0}^{1} f(x, t) g(t) d t \approx K_{M} G_{M \times M} \widehat{P}(x) .
$$

\section{System of Fredholm Integral Equations}

Consider the following coupled system of Fredholm integral equations:

$$
\begin{aligned}
& U(x)+\lambda_{11} \int_{0}^{1} K_{11}(x, t) U(t) d t \\
&+\lambda_{12} \int_{0}^{1} K_{12}(x, t) V(t) d t=f(x), \\
& V(x)+\lambda_{21} \int_{0}^{1} K_{21}(x, t) U(t) d t \\
&+\lambda_{22} \int_{0}^{1} K_{22}(x, t) V(t) d t=g(x),
\end{aligned}
$$

where $\lambda_{11}, \lambda_{12}, \lambda_{21}$, and $\lambda_{22}$ are real constants, $f, g \in$ $C([0,1]), K_{11}, K_{12}, K_{21}, K_{22} \in C([0,1] \times[0,1])$, and $U(x)$, $V(x)$ are unknown functions to be determined. Approximating $U(x)$ and $V(x)$ in terms of Legendre polynomials, we obtain

$$
U(x) \approx H_{M}^{T} \widehat{P}(x), \quad V(x) \approx N_{M}^{T} \widehat{P}(x) .
$$

Using Lemma 1, we have the following approximations:

$$
\begin{aligned}
& \int_{0}^{1} K_{11}(x, t) U(t) d t \approx H_{M}^{T} G_{11} \widehat{P}(x), \\
& \int_{0}^{1} K_{12}(x, t) V(t) d t \approx N_{M}^{T} G_{12} \widehat{P}(x), \\
& \int_{0}^{1} K_{21}(x, t) U(t) d t \approx H_{M}^{T} G_{21} \widehat{P}(x), \\
& \int_{0}^{1} K_{22}(x, t) V(t) d t \approx N_{M}^{T} G_{22} \widehat{P}(x) .
\end{aligned}
$$

Using (18) and (19) in the coupled system (17), we obtain the following system of algebraic equations

$$
\begin{aligned}
& H_{M}^{T} \widehat{P}(x)+\lambda_{11} H_{M}^{T} G_{11} \widehat{P}(x)+\lambda_{12} N_{M}^{T} G_{12} \widehat{P}(x)=F_{1} \widehat{P}(x), \\
& N_{M}^{T} \widehat{P}(x)+\lambda_{21} H_{M}^{T} G_{21} \widehat{P}(x)+\lambda_{22} N_{M}^{T} G_{22} \widehat{P}(x)=F_{2} \widehat{P}(x),
\end{aligned}
$$

which can be written as

$$
\begin{aligned}
& \left(\begin{array}{c}
H_{M}^{T} \widehat{P}(x) \\
N_{M}^{T} \widehat{P}(x)
\end{array}\right)+\left(\begin{array}{c}
\lambda_{11} H_{M}^{T} G_{11} \widehat{P}(x) \\
\lambda_{22} N_{M}^{T} G_{22} \widehat{P}(x)
\end{array}\right) \\
& \quad+\left(\begin{array}{c}
\lambda_{12} N_{M}^{T} G_{12} \widehat{P}(x) \\
\lambda_{21} H_{M}^{T} G_{21} \widehat{P}(x)
\end{array}\right)=\left(\begin{array}{c}
F_{1} \widehat{P}(x) \\
F_{2} \widehat{P}(x)
\end{array}\right) .
\end{aligned}
$$

The transpose of the above system is given by

$$
\begin{aligned}
& \left(H_{M}^{T} \widehat{P}(x) \quad N_{M}^{T} \widehat{P}(x)\right)+\left(\lambda_{11} H_{M}^{T} G_{11} \widehat{P}(x) \quad \lambda_{22} N_{M}^{T} G_{22} \widehat{P}(x)\right) \\
& +\left(\lambda_{12} N_{M}^{T} G_{12} \widehat{P}(x) \lambda_{21} H_{M}^{T} G_{21} \widehat{P}(x)\right) \\
& =\left(F_{1} \widehat{P}(x) \quad F_{2} \widehat{P}(x)\right)
\end{aligned}
$$

which can further be written as

$$
\begin{aligned}
& \left(\begin{array}{ll}
H_{M}^{T} & N_{M}^{T}
\end{array}\right) A+\left(\begin{array}{ll}
H_{M}^{T} & N_{M}^{T}
\end{array}\right)\left(\begin{array}{cc}
\lambda_{11} G_{11} & 0 \\
0 & \lambda_{22} G_{22}
\end{array}\right) A \\
& \quad+\left(\begin{array}{ll}
H_{M}^{T} & N_{M}^{T}
\end{array}\right)\left(\begin{array}{cc}
0 & \lambda_{21} G_{21} \\
\lambda_{12} G_{12} & 0
\end{array}\right) A=\left(\begin{array}{ll}
F_{1} & F_{2}
\end{array}\right) A
\end{aligned}
$$

where

$$
A=\left(\begin{array}{cc}
\widehat{P}(x) & 0 \\
0 & \widehat{P}(x)
\end{array}\right) .
$$

Hence it follows that

$$
\begin{gathered}
\left(\begin{array}{ll}
H_{M}^{T} & N_{M}^{T}
\end{array}\right)+\left(\begin{array}{ll}
H_{M}^{T} & N_{M}^{T}
\end{array}\right)\left(\begin{array}{ll}
\lambda_{11} G_{11} & \lambda_{21} G_{21} \\
\lambda_{12} G_{12} & \lambda_{22} G_{22}
\end{array}\right) \\
-\left(\begin{array}{ll}
F_{1} & F_{2}
\end{array}\right)=0
\end{gathered}
$$

which is a generalized Sylvester type equation and can easily be solved for the unknown $H_{M}$ and $N_{M}$ by any computational software.

\section{Illustrative Examples}

Example 1. Consider the following system of Fredholm integral equation:

$$
\begin{aligned}
U(x) & -\frac{1}{3} \int_{0}^{1}(x+t) U(t) d t-\frac{1}{3} \int_{0}^{1}(x+t) V(t) d t \\
& =\frac{x}{18}+\frac{17}{36} \\
V(x) & -\int_{0}^{1}(x t) U(t) d t-\int_{0}^{1}(x t) V(t) d t \\
& =x^{2}-\frac{19}{12} x+1 .
\end{aligned}
$$

The exact solutions of the system are $U(x)=1+x$ and $V(x)=$ $x^{2}$. The solutions $(U(x), V(x))$ obtained via our technique for 


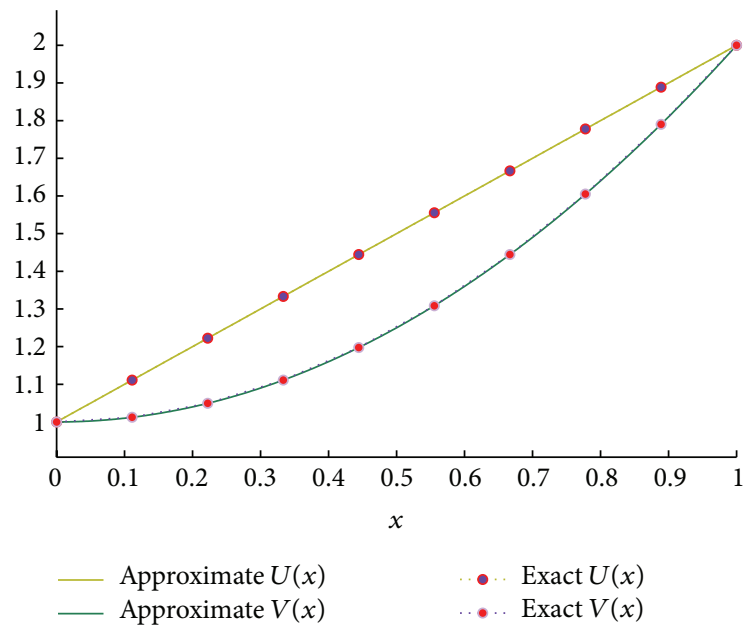

FIGURE 1: Comparison between the exact solutions and the solutions obtained via the new method for $M=3$. Dots represent the exact solution and the approximate solutions are represented by curved lines.

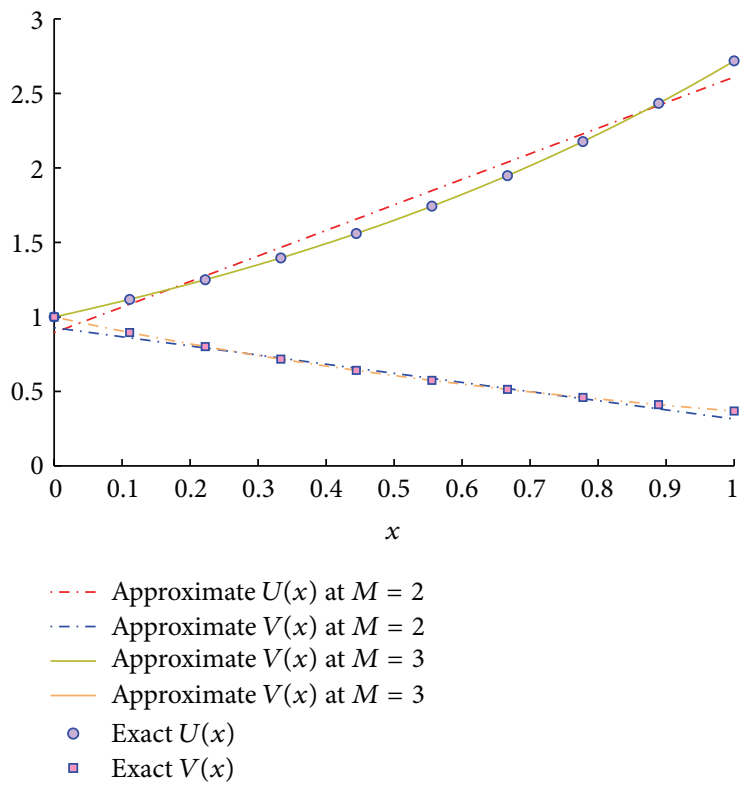

FIGURE 2: Comparing exact solutions with the solutions obtained by our method at different values of $M$.

$M=3$ (small enough) are compared with the exact solutions of the problem in Figure 1, where dots represent the exact solutions and the curves are for the solutions obtained via the new method. From Figure 1, it follows that our solutions matchs very well with the exact solution of the problem even for small value $M$, which shows the effectiveness of our technique.

Example 2. For comparison purposes, consider the following coupled system of Fredholm integral equations:

$$
\begin{aligned}
U(x) & +\int_{0}^{1} e^{(x-t)} U(t) d t+\int_{0}^{1} e^{(x t+2 t)} V(t) d t \\
& =2 e^{x}+\frac{1}{(x+1)}\left(e^{(x+1)}-1\right),
\end{aligned}
$$

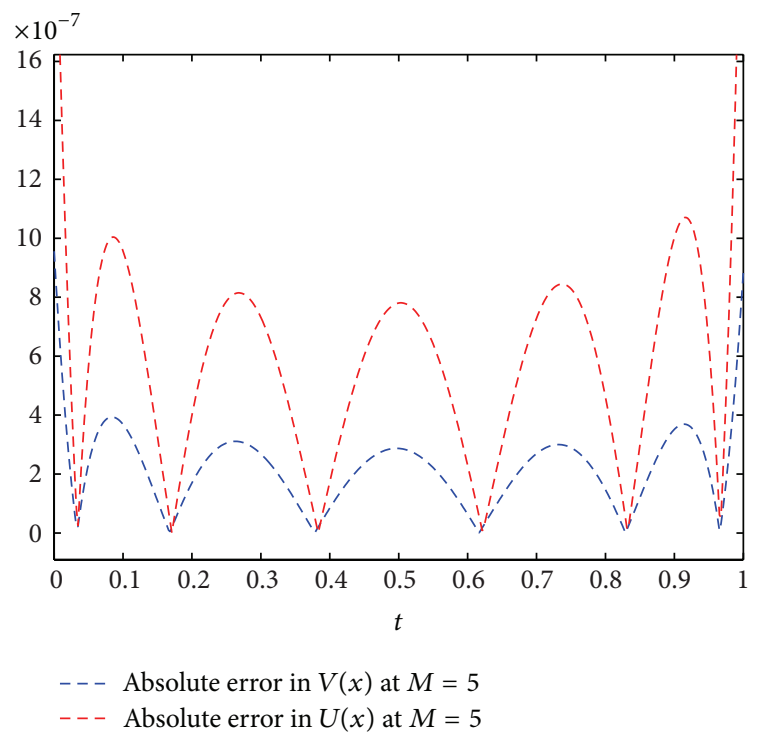

FIgURE 3: Error analysis in $U(x)$ and $V(x)$ for $M=5$.

$$
\begin{aligned}
V(x) & +\int_{0}^{1} e^{(x t)} U(t) d t+\int_{0}^{1} e^{(x+t)} V(t) d t \\
& =e^{x}+e^{-x}+\frac{1}{(x+1)}\left(e^{(x+1)}-1\right) .
\end{aligned}
$$

The exact solutions of the system are $U(x)=e^{x}$ and $V(x)=$ $e^{-x}$. We obtain the approximate solutions of the system for different values of $M$ and compare the results with the exact solutions of the system. For $M=2$ and $M=3$, the comparison is shown in Figure 2, where dots represent the exact solutions of the system and doted curves (red and yellow) represent the approximate solution $(U(x)$ and $V(x))$ obtained via our technique for $M=2$ while Blue and orange dots represent the approximate solutions $(U(x)$ and $V(x))$ obtained via our technique for $M=3$. It is clear that the approximate solutions approach rapidly the exact solutions as the values of $M$ increase. It also shows that the approximate solutions are very close to the exact ones for $M=3$. For example, error of approximation in both $U(x)$ (red doted curve) and $V(x)$ (blue doted curve) is less than $10^{-6}$ for $M=5$ as shown in Figure 3, which is much more acceptable number and demonstrates high accuracy of the new technique. Further, we compare our results with some other available results in the literature. We compare the absolute errors (red line) with the absolute error obtained in [19] using Taylor series approximation and also with absolute error obtained in [16] using numerical solution with block pulses. The results are shown in Figures 4 and 5. From these analyses, it is clear that the absolute error in our method even for small value of $M=4$ is much smaller than those obtained in $[16,19]$ even for much larger values of $m$ such as $m=16,32$. It is a clear indication that the new techniques provide highly accurate solutions as compared to Taylor series approximation method and block pulse method. 


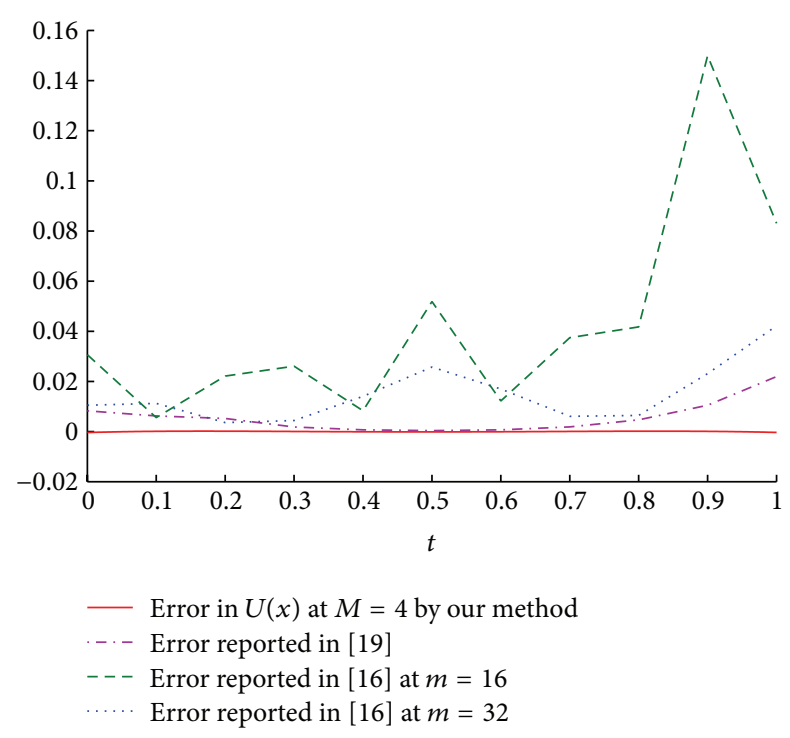

FIgURE 4: Comparing the error estimates in $U(x)$ by our method with error found with Taylor series approximation method (purple dots) and block pulse method (green and blue dots).

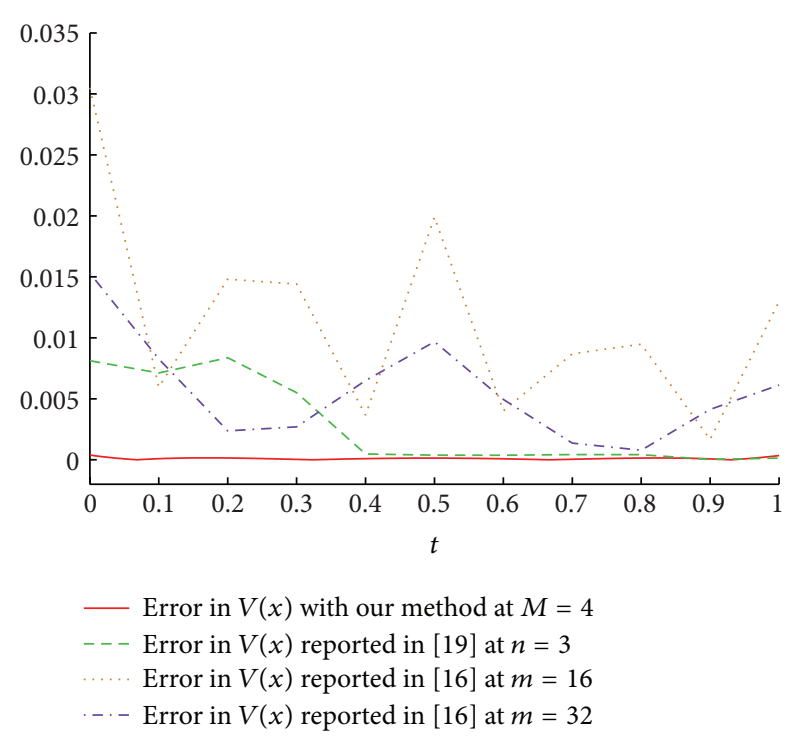

FIGURE 5: Comparing the error estimates in $V(x)$ by our method with error found with taylor series approximation method (green dots) and block pulse method (orange and purple dots).

\section{Conflict of Interests}

The authors declare that there is no conflict of interests regarding the publication of this paper.

\section{References}

[1] T. Triantafyllidis, "Dynamic stiffness of rigid rectangular foundations on the half-space," Earthquake Engineering \& Structural Dynamics, vol. 14, no. 3, pp. 391-411, 1986.

[2] T. Triantafyllidis and B. Prange, "Dynamic subsoil-coupling between rigid rectangular foundations," Soil Dynamics and Earthquake Engineering, vol. 6, no. 3, pp. 164-179, 1987.
[3] P. A. Lewis, "A theory for the diffraction of SH waves by randomly rough surfaces in two dimensions," Quarterly Journal of Mechanics and Applied Mathematics, vol. 49, no. 2, pp. 261-285, 1996.

[4] V. Hutson, "A note on an integral equation occurring in thermoelasticity," Quarterly Journal of Mechanics and Applied Mathematics, vol. 22, no. 1, pp. 125-127, 1969.

[5] P. A. Martin, "On the scattering of elastic waves by an elastic inclusion in two dimensions," Quarterly Journal of Mechanics and Applied Mathematics, vol. 43, no. 3, pp. 275-291, 1990.

[6] K. E. Atkinson, The Numerical Solution of Integral Equation of the Second Kind, Cambridge University Press, Cambridge, Mass, USA, 1997.

[7] R. Kress, Linear Integral Equations, Springer, New York, NY, USA, 1999.

[8] P. K. Kythe and P. Puri, Computational Methods for Linear Integral Equations, Birkhauser, Springer, Boston, Mass, USA, 2002.

[9] P. Linz, Analytical and Numerical Methods for Volterra Equations, SIAM, Philadelphia, Pa, USA, 1985.

[10] B. Alpert, "Wavelet-like bases for the fast solution of secondkind integral equation," SIAM Journal on Scientific Computing, vol. 14, pp. 159-184, 1993.

[11] W. L. Golik, "Wavelet packets for fast solution of electromagnetic integral equations," IEEE Transactions on Antennas and Propagation, vol. 46, no. 5, pp. 618-624, 1998.

[12] E. Babolian, J. Biazar, and A. R. Vahidi, "The decomposition method applied to systems of Fredholm integral equations of the second kind," Applied Mathematics and Computation, vol. 148, no. 2, pp. 443-452, 2004.

[13] J. Biazar, E. Babolian, and R. Islam, "Solution of a system of Volterra integral equations of the first kind by Adomian method," Applied Mathematics and Computation, vol. 139, no. 2-3, pp. 249-258, 2003.

[14] J. Pour-Mahmoud, M. Y. Rahimi-Ardabili, and S. Shahmorad, "Numerical solution of the system of Fredholm integro-differential equations by the Tau method," Applied Mathematics and Computation, vol. 168, no. 1, pp. 465-478, 2005.

[15] A. A. Dascioglu, "Chebesheves polynomial solution of system of linear integral equaiton," Applied Mathematics and Computation, vol. 151, pp. 221-232, 2004.

[16] K. Maleknejad, M. Shahrezaee, and H. Khatami, "Numerical solution of integral equations system of the second kind by Block-Pulse functions," Applied Mathematics and Computation, vol. 166, no. 1, pp. 15-24, 2005.

[17] M. Gülsu and M. Sezer, "Taylor collocation method for solution of systems of high-order linear Fredholm-Volterra integrodifferential equations," International Journal of Computer Mathematics, vol. 83, no. 4, pp. 429-448, 2006.

[18] R. P. Kanwal and K. C. Liu, "A Taylor expansion approach for solving integral equations," International Journal of Mathematical Education in Science and Technology, vol. 20, pp. 411-414, 1989.

[19] Y. Huang, M. Fang, and X.-F. Li, "Approximate solution of a system of linear integral equations by the Taylor expansion method," International Journal of Computer Mathematics, vol. 86, no. 5, pp. 924-937, 2009.

[20] X.-F. Li and M. Fang, "Modified method for determining an approximate solution of the Fredholm-Volterra integral equations by Taylor's expansion," International Journal of Computer Mathematics, vol. 83, no. 8-9, pp. 637-649, 2006. 
[21] K. Maleknejad and N. Aghazadeh, "Numerical solution of Volterra integral equations of the second kind with convolution kernel by using Taylor-series expansion method," Applied Mathematics and Computation, vol. 161, no. 3, pp. 915-922, 2005.

[22] Y. Ren, B. Zhang, and H. Qiao, "A simple Taylor-series expansion method for a class of second kind integral equations," Journal of Computational and Applied Mathematics, vol. 110, no. 1, pp. 15-24, 1999.

[23] M. Sezer, "Taylor polynomial solution of Volterra integral equations," International Journal of Mathematical Education in Science and Technology, vol. 25, pp. 625-633, 1994.

[24] B.-Q. Tang and X.-F. Li, "Approximate solution to an integral equation with fixed singularity for a cruciform crack," Applied Mathematics Letters, vol. 21, no. 12, pp. 1238-1244, 2008.

[25] S. Yalçinbaş and M. Sezer, "The approximate solution of highorder linear Volterra-Fredholm integro-differential equations in terms of Taylor polynomials," Applied Mathematics and Computation, vol. 112, no. 2-3, pp. 291-308, 2000.

[26] A. Saadatmandi and M. Dehghan, "A new operational matrix for solving fractional-order differential equations," Computers and Mathematics with Applications, vol. 59, no. 3, pp. 1326-1336, 2010.

[27] S. Nemati and Y. Ordokhani, "Legendre expansion methods for the numerical solution of nonlinear 2D fredholm integral equations of the second kinds," Journal of Applied Mathematics \& Informatics, vol. 31, pp. 609-621, 2013. 


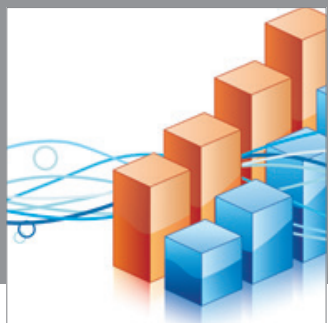

Advances in

Operations Research

mansans

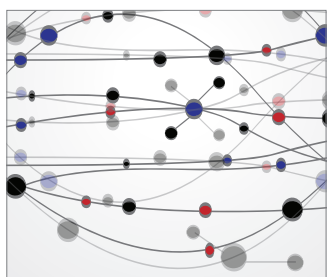

The Scientific World Journal
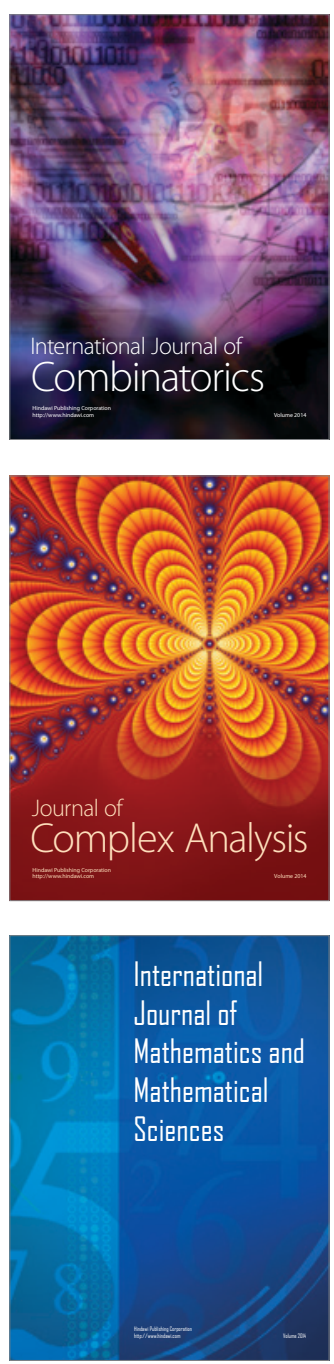
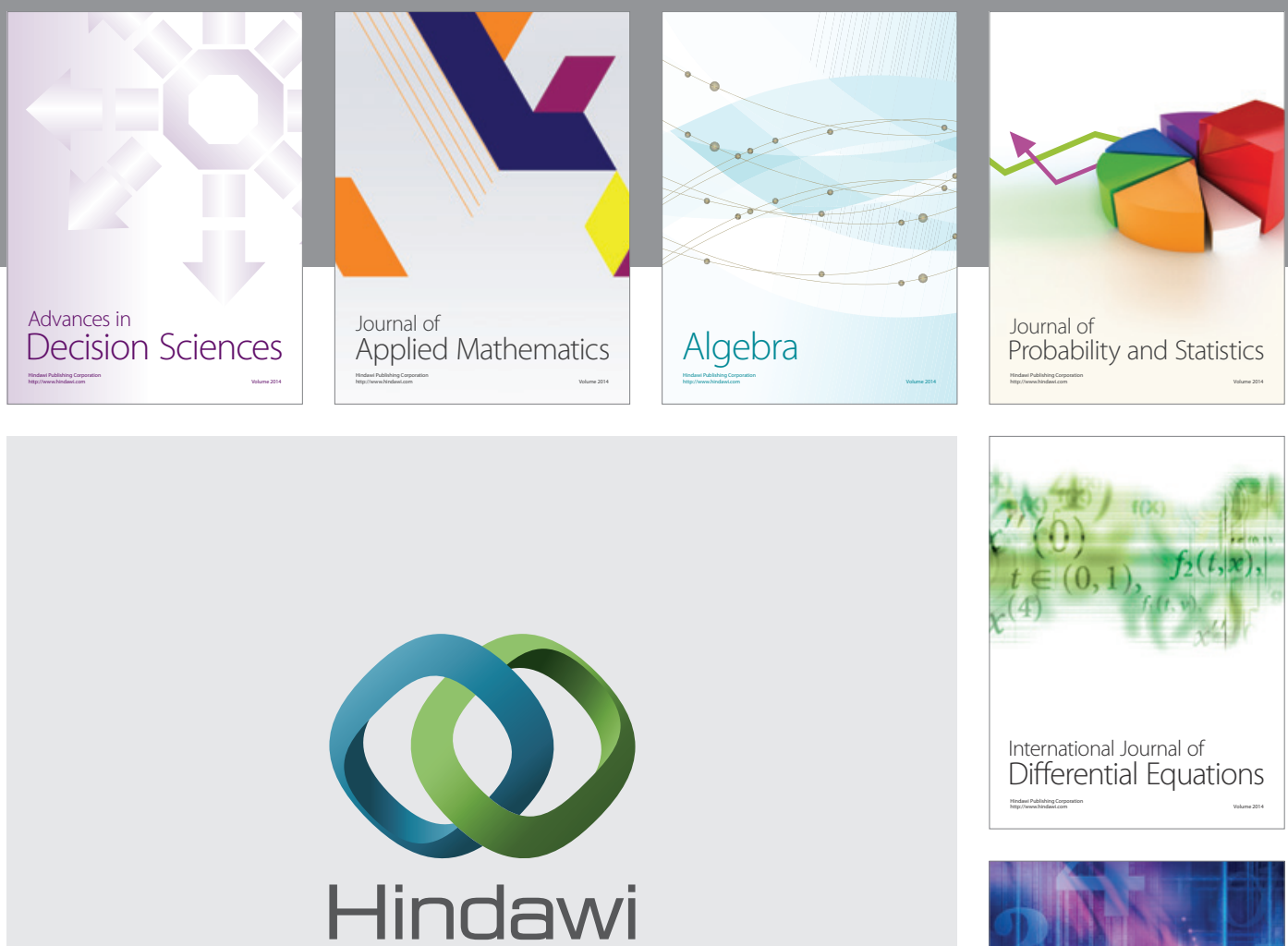

Submit your manuscripts at http://www.hindawi.com
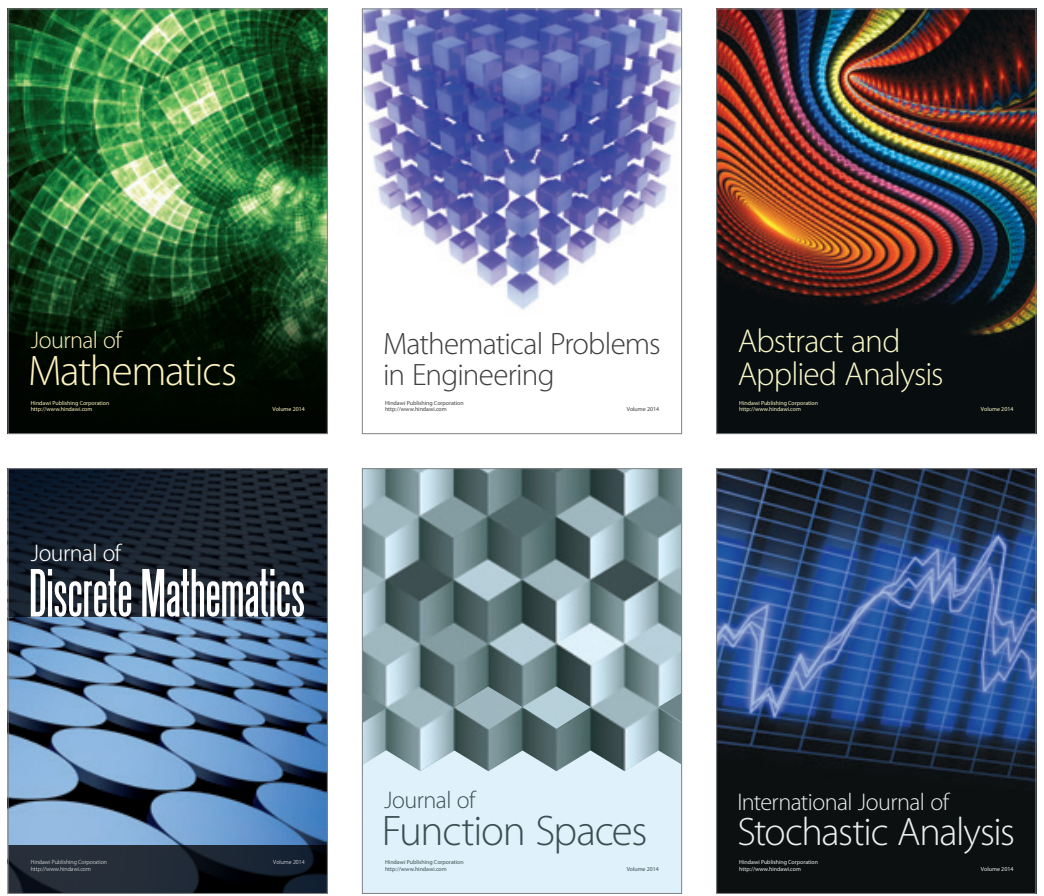

Journal of

Function Spaces

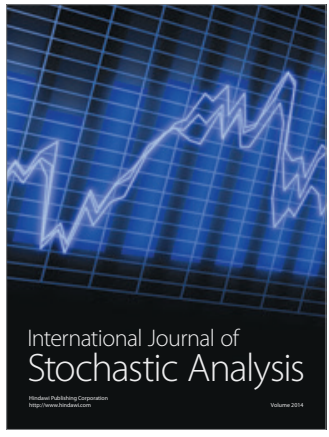


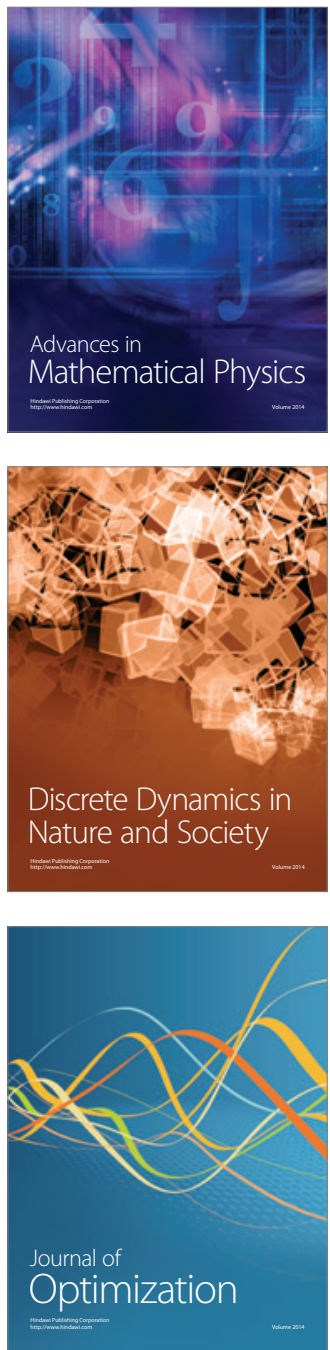